\title{
Anita K. Simonds (Royal Brompton \& Harefield NHS Trust, UK) and Wilfried de Backer (University of Antwerp, Belgium). ERS handbook of respiratory sleep medicine
}

\author{
Hermes ISBN: 978-1-84984-023-1
}

\author{
Kingman Strohl
}

Received: 26 June 2013 / Accepted: 19 July 2013 / Published online: 18 February 2014

(C) Springer-Verlag Berlin Heidelberg 2014

This is a mid-level content book of knowledge in the area of sleep medicine. It is mid-level because it is neither a comprehensive nor an exhaustive review, and not a low-level outline of the topics. It was intended to complement the sleep HERMES task force report of the European Respiratory Society and to prepare trainees in respiratory medicine for European examinations. Most authors are the "pioneers" in respiratory disorders of sleep who learned "on the fly" to recognize the importance of co-morbid and primary sleep disorders. Along with concise descriptions of causes and treatments of sleep disordered breathing, there are presented equally valuable content areas, euphemistically called "non-respiratory sleep disorders" in the USA, content which is smart, informative, and up-to-date.

The handbook literally fits in your hand, and is placed easily in a large side pocket of a physician coat or beside the computer. There are nine general areas, and in each, there is a discussion of prototypic disorders and some alternative diagnosis according to disease (several on respiratory disorders) or major presenting symptoms (hypersomnolence). The strengths are those of diagnostic recognition of most all of the disorders presented, and treatments of respiratory failure, respiratory diseases, and sleep-related breathing disorders- diagnosis, epidemiology, treatments, and outcomes. There is less relatively less depth on the non-respiratory disorders of sleep, but very passable and informative for present training and practice. The general area of "circadian disorders" could better articulate the need to apply chronopharmacologic principles to disease processes and drug administration in respiratory practice. If you are in the active practice of respiratory medicine or dental sleep medicine, there is enough here to lead to an informed differential diagnosis, discussion and plan initial therapy for something like Restless Legs Syndrome or REM Behavior Disorder, and referral to another specialist which would benefit from knowledge of your thinking and expected outcome for the visit. The book has face validity as well, for the presentations suggest that the authors have attended in clinics or worked-up patients on a regular basis.

Towards its intended use, this is a "value" read for a fellow or physician in a non-sleep specialist program who needs to review "sleep" for specialty or continuing certification examinations. I also recommend this book for those who need a handy reference to the diagnosis and approach to sleep disorders from any adult medical, dental, or surgical field, not only pulmonary for non-respiratory issues but neurology, psychiatry, etc. for descriptions of respiratory disorders. In the past 2 months, as a specialist, I have used it to "fact check" a presentation to sleep fellows on insomnia and a presentation for a psychiatry training program.
K. Strohl $(\bowtie)$

Department of Medicine, Veterans Administration Medical Center 111J (W), Case Western Reserve University, 10701 East Boulevard, Cleveland, OH 44106, USA

e-mail: kpstrohl@aol.com 